\title{
The occurrence and characterization of Campylobacter jejuni and $C$. coli in organic pigs and their outdoor environment
}

\author{
A.N. Jensen $^{\text {a,* }}$, A. Dalsgaard ${ }^{\text {b }}$, D.L. Baggesen ${ }^{\text {a }}$, E.M. Nielsen ${ }^{c}$ \\ a Danish Institute for Food and Veterinary Research, Department of Microbiological Food Safety, \\ Bülowsvej 27, DK-1790 Copenhagen V, Denmark \\ ${ }^{\mathrm{b}}$ Department of Veterinary Pathobiology, The Royal Veterinary and Agricultural University, Copenhagen, Denmark \\ ${ }^{\mathrm{c}}$ Statens Serum Institut, Copenhagen, Denmark
}

Received 22 December 2005; received in revised form 3 March 2006; accepted 10 March 2006

\begin{abstract}
The occurrence and species distribution of thermophilic Campylobacter was investigated in organic outdoor pigs. An increased exposure of outdoor pigs to $C$. jejuni from the environment may cause a shift from a normal dominance of $C$. coli to more $C$. jejuni, which may imply a concern of reduced food safety.

Bacteriological methods for determination of Campylobacter excretion level were combined with colony-blot hybridization and real-time PCR for specific detection of $C$. jejuni in pigs. Campylobacter was isolated from pigs $(n=47)$, paddock environment $(n=126)$ and wildlife $(n=44)$, identified to species by real-time PCR and sub-typed by serotyping (Penner) and pulse-field gel electrophorsis (PFGE) genotyping.

All pigs excreted Campylobacter $\left(10^{3}-10^{7} \mathrm{CFU} \mathrm{g} \mathrm{g}^{-1}\right.$ faeces) from the age of 8-13-weeks old. C. jejuni was found in $29 \%$ of pigs in three consecutive trials and always in minority to C. coli $(0.3-46 \%)$. C. jejuni and C. coli were isolated from $10 \%$ and $29 \%$ of the environmental samples, respectively, while crow-birds and rats harboured $C$. jejuni. Individual pigs hosted several strains (up to nine serotypes). The paddock environment was contaminated with $C$. coli serotypes similar to pig isolates, while most of the $C$. jejuni serotypes differed. $C$. jejuni isolates of different origin comprised few similar serotypes, just one identical genotype was common between pigs, environment and birds.

In conclusion, the occurrence of $C$. jejuni varied considerably between the three groups of outdoor pigs. Furthermore, transfer of $C$. jejuni to the outdoor pigs from the nearby environment was not predominant according to the subtype dissimilarities of the obtained isolates.
\end{abstract}

(C) 2006 Elsevier B.V. All rights reserved.

Keywords: Thermophilic Campylobacter; Strain diversity; Penner-serotyping; PFGE; Wildlife

* Corresponding author. Tel.: +45 72346328; fax: +45 72346001 .

E-mail address: anj@dfvf.dk (A.N. Jensen).

\section{Introduction}

Campylobacter jejuni is the dominant cause of human cases of campylobacteriosis (Nielsen et al., 
1997; Anon., 2004). Pigs seem to be a natural reservoir of Campylobacter spp. with a prevalence between $50 \%$ and $100 \%$ and excretion levels ranging from $10^{2}$ to $10^{7} \mathrm{CFU} / \mathrm{g}$, but opposite to most other animals, pigs show a dominance of $C$. coli (Munroe et al., 1983; Manser and Dalziel, 1985; Nielsen et al., 1997; Alter et al., 2005; Boes et al., 2005). Nevertheless, American studies found that $C$. jejuni may constitute a majority (up to 87\%) of the Campylobacter spp. detected on hog farms (Harvey et al., 1999; Young et al., 2000). A high prevalence of $C$. jejuni has also been reported for porcine livers (Kramer et al., 2000).

$C$. jejuni may co-exist with $C$. coli in pigs, but are typically present in 10-100-fold lower numbers than C. coli (Madden et al., 2000; Jensen et al., 2005). Detection of $C$. jejuni present in minority to $C$. coli is difficult by conventional picking of a few colonies and discrimination between $C$. coli and $C$. jejuni is problematic due to hippurate-negative $C$. jejuni (Totten et al., 1987; Jensen et al., 2005). Application of alternative molecular methods, like real-time PCR and colony-blot hybridization, will aid to avoid underestimation of the presence of $C$. jejuni in pigs (Jensen et al., 2005).

The closer contact of outdoor pigs to the environment and wildlife, where $C$. jejuni predominates (Kapperud and Rosef, 1983; Manser and Dalziel, 1985; Petersen et al., 2001; Waldenstrom et al., 2002; Brown et al., 2004), may cause a shift towards more $C$. jejuni. This could be of potential food safety concern, because $C$. jejuni is the major cause of campylobacteriosis in humans.

Investigations on the dynamics of Campylobacter infections in organic outdoor pig production systems are scarce. However, free-range pigs from a single organic farm seemed to be colonized with Campylobacter earlier in life than conventional pigs (Alter et al., 2005). Outdoor organic pigs may differ from conventional pigs with respect to the occurrence of Campylobacter. For example, the lower animal density probably reduces the infection pressure and roughage stimulates the intestinal flora, which is likely to reduce the susceptibility to infections (Mikkelsen et al., 2004).

The overall aim of the current study was to investigate the temporal infection dynamics of natural Campylobacter infections in organic pigs raised outdoor. Specifically, we wanted to examine the $C$. jejuni and C. coli species distribution in groups and individual pigs; to determine the excretion level of Campylobacter spp.; the potential interaction with the environment including wildlife.

\section{Materials and methods}

\subsection{Sampling}

Rectal faecal samples from 47 organic raised pigs obtained from a single farm were collected once per week from shortly after weaning at the age of 7- until 13-weeks old. The study was divided in three trials that were initiated in late April, mid-June and late July 2003 including 16, 16 and 15 pigs in each trial, respectively. The pigs were held in two outdoor paddocks, referred to as A and B, at a university research farm in Taastrup, Denmark. The experiments were performed in accordance with the regulations for organic pig production, e.g. with respect to animal density, access to wallowing area and organic feed including roughage. The pigs used for this study were included as Salmonellanegative control pigs in a Salmonella infection study described elsewhere (Jensen et al., 2006).

Soil and water samples from paddock A and B were collected four, two and three times in each trial, respectively. The first sampling took place prior to the introduction of pigs into the paddocks. Water $(50 \mathrm{ml})$ was collected from the water drinking cup and soil $(>30 \mathrm{~g}$ ) was collected as pools of small samples of surface soil $(1-5 \mathrm{~cm})$ from six different locations in the paddock.

Furthermore, rodents $(n=21)$ and shrews $(n=1)$ were caught in traps around the paddocks for a period of 2 weeks in late August and in addition, 22 birds were shot or caught as previously described (Jensen et al., 2004).

To avoid cross-contamination when collecting the different sample types, disposable or disinfected equipment was used for collecting samples along with good hygiene practices.

\subsection{Enumeration and isolation of Campylobacter spp.}

\subsubsection{Faeces}

The faecal samples were kept on ice during transport to the laboratory and stored at $4{ }^{\circ} \mathrm{C}$ until 
testing the next day. Ten-fold dilution series of faeces $(1 \mathrm{~g})$ were cultured by direct plating on modified charcoal-cefoperazone-deoxycholate agar plates (mCCDA) (Campylobacter Blood Free Selective Agar Base (Oxoid, Basingstoke, UK) with CCDA Selective Supplement (Oxoid, SE155E)) and from every second sampling week, the sample dilutions were, in addition to the direct plating, enriched in Bolton Broth (Bolton broth without blood, prepared according to the recommendations of the Bacteriological Analytical Manual Online) as previously described (Jensen et al., 2005). After incubation of mCCDA plates $\left(48 \mathrm{~h}\right.$ at $41.5^{\circ} \mathrm{C}$; microaerobic conditions), Campylobacter spp. colonies (based on colony and cell morphology by microscopy) from direct plating were counted (detection limit $>100 \mathrm{CFU} \mathrm{g}^{-1}$ ). Five colonies from Campylobacter-positive samples were sub-cultured three times on blood agar (BA) to obtain pure cultures, then identified and stored $\left(15 \%\right.$ glycerol broth, $\left.-80{ }^{\circ} \mathrm{C}\right)$ for further characterization. The identification was based on hippurate hydrolysis (specific for $C$. jejuni) and a species specific real-time PCR developed for $C$. jejuni, C. coli, C. lari and C. upsaliensis (Jensen et al., 2005).

\subsubsection{Environmental samples}

The environmental samples were transported at ambient temperature to the laboratory and stored at $4{ }^{\circ} \mathrm{C}$ until testing the next day. Homogenized soil samples $(5 \mathrm{~g})$ were added to Bolton broth in a ratio of $1: 9$, and water samples $(50 \mathrm{ml})$ were filtrated onto a $0.45 \mu \mathrm{m}$ membrane filter (Millipore, Billerica, MA, USA) by use of vacuum and filters were added to $9 \mathrm{ml}$ of Bolton broth for pre-enrichment, diluted and plated on mCCDA as described above. When possible, five colonies of Campylobacter spp. were sub-cultured, identified and stored as described above.

\subsubsection{Wildlife}

The rodents and birds were stored at $4{ }^{\circ} \mathrm{C}$ up to 3 days before dissected. The presence of Campylobacter spp. was examined qualitatively by streaking a cotton swab with the homogenized intestinal set (stomached $2 \times 30 \mathrm{~s}$ ) onto two mCCDA plates and incubated for $48 \mathrm{~h}$ at $41.5^{\circ} \mathrm{C}$ under microaerobic conditions. Two isolates were obtained from each Campylobacter-positive sample as described above.

\subsection{Detection of $C$. jejuni in pigs}

The pigs were examined for co-colonization of $C$. jejuni and $C$. coli by application of a colony-blot hybridization method based on the hipO gene specific for $C$. jejuni, previously evaluated and described (Jensen et al., 2005). For all samples $(n=261)$, hybridization was performed on colonies obtained by direct plating of dilution series on mCCDA plates (plate with most single colonies, i.e. typically 50-150 colonies per plate). An additional hybridization approach was applied for faecal samples from every second sampling week $(n=134)$ to enhance the sensitivity of the assay and to compare the capacity of each approach to detect $C$. jejuni in naturally infected samples. For these samples, the Bolton enrichment broth dilutions were first screened for $C$. jejuni by rtPCR (Jensen et al., 2005) and then the positive samples were diluted and spread on mCCDA plates. Colony-blot hybridization was performed after two days incubation. When presumptive $C$. jejuni colonies were detected by colony-blot hybridization, cell material from the corresponding colony on the mCCDA plate was picked (up to five colonies) and sub-cultured to obtain bacterial isolates for further characterization by serotyping and PFGE typing (Jensen et al., 2005). A pig was considered $C$. jejuni-positive when an isolate was confirmed as $C$. jejuni irrespective of the detection approach.

\subsection{Serotyping}

The Campylobacter isolates were serotyped according to the 'Penner' scheme for heat-stable serotyping (Penner et al., 1983). The production of antisera, dilutions of antisera and interpretation of reactions were described previously (Nielsen et al., 1997). The Campylobacter isolates were serotyped in both $C$. coli and $C$. jejuni sets of antisera (19 and 47 antisera, respectively), independent on their species. All the C. jejuni isolates from faecal and environmental samples (one to seven isolates per sample) were serotyped. A minimum of five $C$. coli from $C$. jejuni-positive faecal samples and one to two $C$. coli from environmental samples were serotyped. 


\subsection{Pulse field gel electrophoresis typing}

Selected isolates of $C$. jejuni serotypes found in more than one type of sample source were analysed by PFGE for further discrimination between isolates. Only one isolate of each serotype per C. jejunipositive animal and environmental sample were PFGE typed. PFGE was performed as described by Ribot et al. (2001). The DNA was digested with the restriction enzyme SmaI and a CHEF Mapper (BioRad Laboratories, Herlev, Denmark) was used to separate the fragments (initial switch time $6 \times 75 \mathrm{~s}$, final switch time $38 \times 35 \mathrm{~s}$ and running time $19 \mathrm{~h}$ ). A difference in one or more band locations or the number of bands was considered to represent different PFGE types.

\section{Results}

\subsection{Campylobacter spp. excretion level in pigs}

All organic pigs $(n=47)$ excreted thermophilic Campylobacter spp. at all samplings during the 6 weeks of study from the age of 8 to 13 weeks. The level of Campylobacter spp. ranged from approximately $10^{3}$ to $10^{7} \mathrm{CFU} \mathrm{g}{ }^{-1}$ faeces (geometric mean $2.3 \times 10^{5} \mathrm{CFU} \mathrm{g}^{-1}$ ) with no clear temporal variations.

\subsection{Detection of $C$. jejuni and C. coli in organic raised pigs}

The pigs were examined for co-colonization of $C$. jejuni and $C$. coli by application of a colony-blot hybridization method and the distribution between the two species was calculated as the ratio between the $C$. jejuni target-probe hybrids and the total number of Campylobacter. A majority of the obtained isolates was identified as C. coli, while a total of 107 C. jejuni isolates were obtained from 25 faecal samples representing 14 pigs $(29.8 \%)$ (Table 2). However, there was a big variation between the three trials carried out successively from April to September, as $18.8 \%, 0 \%$ and $78.6 \%$ of the pigs were C. jejunipositive, respectively. $C$. jejuni was detected from one to four out of the six samplings with an average of 1.8 times, but not necessarily in consecutive weeks (Table 2). The percentage of $C$. jejuni colonies out of total number of Campylobacter colonies ranged from $1 / 300(0.3 \%)$ to $89 / 192(46.4 \%)$ with an average of $6.7 \%$ (265/3947). This shows that C. jejuni was present in minority to other Campylobacter and indicates the potential difficulties in detection and isolation $C$. jejuni by the normal picking of a single colony from an agar plate.

\subsection{Detection of Campylobacter in the environment}

A total of 126 surface soil $(n=108)$ and water $(n=18)$ samples from the paddock environment were examined for the presence of Campylobacter during the three trials. Isolates were obtained from a total of 44 samples (34.9\%) with C. coli found in 15, 9 and 12 samples (29\% of samples) and C. jejuni in 5, 3 and 5 samples (10\% of samples) in each period (Table 1). Both species were isolated in five samples. Examination for Campylobacter spp. in wildlife resulted in detection of $C$. jejuni in rats ( $2 / 2 C$. jejuni positive) and crowbirds including jackdaws, magpies and crows (18/19 C. jejuni positive), but not in shrews and mice $(0 / 20$ C. jejuni positive) or in lark and great tit $(0 / 3 C$. jejuni positive). A total of $44 C$. jejuni isolates from wildlife were obtained.

\subsection{Serotyping}

\subsubsection{Pig isolates}

A total of 107 C. jejuni isolates from the 14 C. jejunipositive pigs were serotyped. They comprised five different serotypes, with detection of the serotypes O:23.36 (74 isolates in total) in nine pigs, O:1.44 (16 isolates in total) in three, O:2 (15 isolates in total) in five and O:33 and O:52 in one pig. The serotype O:23.36 was only isolated in the third period. Apparently, most pigs hosted a single $C$. jejuni serotype. However, four pigs in the third trial harboured O:23.36 in addition to one (O:2 or O:1.44) or two (O:33 and O:52) other strains. In two cases these strains was isolated from the same faecal sample, however, the cocolonization of $C$. jejuni was not consistent over time (Table 2).

A total of 141 C. coli isolates from the 14 C. jejunipositive pigs were serotyped. The serotypes O:11 $(n=27)$ and 0:6.7 $(n=23)$ were each isolated from 11 pigs and the O:4-complex $(n=1)$ or O:4.34-complex 
Table 1

Detection of $C$. jejuni in organic outdoor pigs ${ }^{\mathrm{a}}$ and their paddock environment ${ }^{\mathrm{b}}$

\begin{tabular}{|c|c|c|c|c|c|c|}
\hline \multirow{3}{*}{$\begin{array}{l}\text { Age of pigs } \\
\text { (weeks) }\end{array}$} & \multicolumn{6}{|c|}{ No. of $C$. jejuni-positive faecal and environmental samples } \\
\hline & \multicolumn{2}{|l|}{ Trial 1} & \multicolumn{2}{|l|}{ Trial 2} & \multicolumn{2}{|l|}{ Trial 3} \\
\hline & $\begin{array}{l}\text { Pigs } \\
(n=16)\end{array}$ & $\begin{array}{l}\text { Environment } \\
(n=14)\end{array}$ & $\begin{array}{l}\text { Pigs } \\
(n=16)\end{array}$ & $\begin{array}{l}\text { Environment } \\
(n=14)\end{array}$ & $\begin{array}{l}\text { Pigs } \\
(n=15)\end{array}$ & $\begin{array}{l}\text { Environment } \\
(n=14)\end{array}$ \\
\hline 8 & 0 & 0 & 0 & 1 & 4 & 2 \\
\hline 9 & 0 & 1 & $0^{\mathrm{c}}$ & n.d. & $1^{\mathrm{c}}$ & n.d. \\
\hline 10 & $0^{\mathrm{c}}$ & n.d. ${ }^{\mathrm{d}}$ & $0^{\mathrm{c}}$ & n.d. & 3 & 0 \\
\hline 11 & 0 & 4 & $0^{\mathrm{c}}$ & n.d. & $5^{\mathrm{c}}$ & n.d. \\
\hline 12 & $1^{\mathrm{c}}$ & n.d. & $0^{\mathrm{c}}$ & n.d. & $4^{\mathrm{c}}$ & n.d. \\
\hline 13 & 2 & 0 & 0 & 2 & 5 & 3 \\
\hline Total no. of samples & 96 & 56 & 96 & 28 & 90 & 42 \\
\hline$\%$ C. jejuni-positive & 3 & 9 & 0 & 11 & 24 & 12 \\
\hline
\end{tabular}

a A total of 47 pigs were examined once per week from the age of 8-13-weeks old in three consecutive trials.

${ }^{\mathrm{b}}$ Seven soil and water samples were collected weekly from the environment of paddocks A and B.

${ }^{c}$ In this week, the detection of $C$. jejuni is based only on colony-blot hybridization on non-enriched samples.

${ }^{\mathrm{d}}$ n.d.: not done.

Table 2

Occurrence of heat-stable serotypes ${ }^{\mathrm{a}}$ (Penner) among C. jejuni isolates from organic pigs and environment ${ }^{\mathrm{b}}$ in two outdoor paddocks (A and B) in three consecutive trials (1-3)

\begin{tabular}{|c|c|c|c|c|c|c|c|c|}
\hline \multirow[t]{2}{*}{ Trial } & \multirow[t]{2}{*}{ Paddock } & \multirow[t]{2}{*}{ Pig } & \multicolumn{6}{|c|}{ Detection of $C$. jejuni (serotypes) (sampling week) } \\
\hline & & & Week 1 & Week 2 & Week 3 & Week 4 & Week 5 & Week 6 \\
\hline 1 & $\mathrm{~A}$ & 5 & - & - & - & - & - & $2^{c}$ \\
\hline 1 & A & 6 & - & - & - & - & - & $2^{\mathrm{c}}$ \\
\hline 1 & $\mathrm{~B}$ & 3 & - & - & - & - & $1.44^{\mathrm{c}}$ & - \\
\hline 3 & A & 1 & - & - & - & $2,23.36^{\mathrm{d}}$ & - & - \\
\hline 3 & $\mathrm{~A}$ & 3 & - & - & - & - & - & $1.44^{\mathrm{c}}$ \\
\hline 3 & A & 5 & - & - & - & 23.36 & $23.36^{\mathrm{d}}$ & 2 \\
\hline 3 & $\mathrm{~A}$ & 6 & $23.36^{\mathrm{d}}$ & - & - & - & - & $1.44^{\mathrm{c}}$ \\
\hline 3 & $\mathrm{~B}$ & 1 & $23.36^{\mathrm{d}}$ & - & - & - & $23.36^{\mathrm{d}}$ & - \\
\hline 3 & B & 2 & & - & $23.36^{\mathrm{d}}$ & - & - & - \\
\hline 3 & B & 3 & - & - & - & $23.36^{\mathrm{d}}$ & $23.36^{\mathrm{d}}, 33,52$ & - \\
\hline 3 & B & 4 & - & - & - & $2^{c}$ & - & - \\
\hline 3 & B & 5 & - & - & $23.36^{\mathrm{d}}$ & $23.36^{\mathrm{d}}$ & 23.36 & 23.36 \\
\hline 3 & B & 6 & $23.36^{\mathrm{d}}$ & $23.36^{\mathrm{d}}$ & 23.36 & - & - & 23.36 \\
\hline 3 & B & 7 & $23.36^{\mathrm{d}}$ & - & - & - & - & - \\
\hline 1 & $\mathrm{~A}^{\mathrm{b}}$ & & - & 5,55 & n.d. & 47, NT & n.d. & - \\
\hline 1 & $\mathrm{~B}^{\mathrm{b}}$ & & - & - & n.d. & $1.44^{\mathrm{c}}, \mathrm{NT}$ & n.d. & - \\
\hline 2 & $\mathrm{~A}^{\mathrm{b}}$ & & $33, \mathrm{NT}$ & n.d. & n.d. & n.d. & n.d. & $33,37.56,38$ \\
\hline 2 & $\mathrm{~B}^{\mathrm{b}}$ & & - & n.d. & n.d. & n.d. & n.d. & $2^{\mathrm{c}}$ \\
\hline 3 & $\mathrm{~A}^{\mathrm{b}}$ & & $2,33, \mathrm{NT}$ & n.d. & - & n.d. & n.d. & $2,4 \mathrm{c}, 27,33, \mathrm{NT}$ \\
\hline 3 & $\mathrm{~B}^{\mathrm{b}}$ & & 35 & n.d. & - & n.d. & n.d. & 41,52 \\
\hline
\end{tabular}

${ }^{\text {a }}$ NT, non-typeable; c, complex; -, no C. jejuni; n.d., not done (no sampling).

${ }^{b}$ Isolates obtained from any of the seven different locations in the paddock environment.

${ }^{c}$ Identical PFGE profile within each serotype.

${ }^{\mathrm{d}}$ Identical PFGE profile in the 13 tested strains. 
Table 3

Occurrence of $C$. coli serotypes $^{\text {a }}$ (Penner) in individual pigs (C. jejuni positive)

\begin{tabular}{|c|c|c|c|c|c|c|c|c|c|}
\hline \multirow[t]{2}{*}{ Trial } & \multirow[t]{2}{*}{ Paddock } & \multirow[t]{2}{*}{ Pig } & \multirow[t]{2}{*}{ Isolates $^{\mathrm{b}}(n)$} & \multicolumn{6}{|c|}{ C. coli serotypes (sampling week) } \\
\hline & & & & Week 1 & Week 2 & Week 3 & Week 4 & Week 5 & Week 6 \\
\hline 1 & A & 5 & 9 & $6.7,51.54$ & NT & n.d. & n.d. & n.d. & $24,4.34 \mathrm{c}, 51.54$ \\
\hline 1 & A & 6 & 11 & 6.7 & $4.34 \mathrm{c}, 51.54$ & 6.7 & n.d. & n.d. & $4.34 c, 46$ \\
\hline 1 & $\mathrm{~B}$ & 3 & 8 & $4.34 \mathrm{c}$ & $4.34 \mathrm{c}$ & $4.34 \mathrm{c}, 11$ & n.d. & n.d. & $5,6.7$ \\
\hline 3 & A & 1 & 6 & 30,48 & n.d. & 11,48 & n.d. & n.d. & 30 \\
\hline 3 & A & 3 & 5 & $6.7,30$ & n.d. & 11,30 & n.d. & n.d. & 26.34 \\
\hline 3 & A & 5 & 8 & $6.7,48$ & n.d. & 6.7 & n.d. & n.d. & 11,48 \\
\hline 3 & A & 6 & 17 & $6.7,11,48$ & n.d. & 11 & n.d. & $4 c, 49$ & $11,30,48, \mathrm{NT}$ \\
\hline 3 & B & 1 & 12 & $20,26.34$ & n.d. & $6.7,11,26.34$ & n.d. & $6.7,49$ & $20,51.54$ \\
\hline 3 & B & 2 & 8 & 11 & n.d. & $11,26.34,30$ & n.d. & n.d. & $11,4.34 \mathrm{c}$ \\
\hline 3 & B & 3 & 6 & 6.7 & n.d. & 48 & n.d. & 46 & $6.7,48$ \\
\hline 3 & B & 4 & 5 & 20,30 & n.d. & 11 & n.d. & n.d. & 6.7 \\
\hline 3 & $\mathrm{~B}$ & 5 & 20 & 11,46 & n.d. & $11,20,34,48, \mathrm{NT}$ & 11 & $20,4.34 \mathrm{c}$ & 30,46 \\
\hline 3 & $\mathrm{~B}$ & 6 & 18 & $6.7,11$ & $26.34,30,48$ & $6.7,48$ & n.d. & n.d. & $4.34 \mathrm{c}, 11,24$ \\
\hline 3 & B & 7 & 8 & 11 & n.d. & 51.54 & n.d. & n.d. & $5,6.7$ \\
\hline
\end{tabular}

${ }^{a}$ Serotypes: NT, non-typeable; c, complex, n.d., not done.

${ }^{\mathrm{b}} n$ : total number of serotyped isolates.

$(n=16)$ from 7 pigs (Table 3). These serotypes are considered typical $C$. jejuni serotypes, however, these isolates were clearly identified as $C$. coli by rt-PCR (also hippurate negative) (Jensen et al., 2005). The other serotypes shown by the $C$. coli isolates were the typical $C$. coli serotypes including O:30 (seven pigs), O:48 (six pigs), O:51.54 (four pigs), O:26.34 (four pigs), O:20 (three pigs), O:46 (three pigs), O:5 (two pigs), O:49 (two pigs), O:24 (two pigs) and O:34 (one pig). Four isolates were non-typeable. Up to eight different $C$. coli serotypes were found in individual pigs.

\subsubsection{Environmental isolates}

A total of 75 C. coli and 79 C. jejuni isolates from the paddock environment were serotyped. Table 2 shows an overview of the different serotypes of $C$. jejuni found during the three trials in the pigs and the paddock environment, respectively. Serotyping revealed an overlap of the $C$. jejuni serotypes O:2, O:1.44, O:33 and O:52 between pigs and environment, while the most common $C$. jejuni serotype in the pigs, O:23.36, never was recovered from the environment but in one of the rats only (see below). The other $C$. jejuni isolates found in the environment included O:4.34-complex, O:5, O:27, O:35, O:37.56, O:38, O:41, O:47 and O:55. Six of these serotypes were not shown by Campylobacter strains originating from either pigs or wildlife. The C. coli isolates from the environment comprised the serotypes O:6.7, O:11, O:20, O:30, O:46, O:48, O:49, O:51.54, O:54, O:4.34complex and non-typeable (NT). All C. coli serotypes found in the environment were also found in the pigs, but three serotypes isolated from pigs were never recovered from the paddock environment.

A total of 44 C. jejuni isolates from wildlife were serotyped and for approximately half of the animals the two isolates obtained from the same animal were of identical serotype. The $C$. jejuni isolates included the serotypes O:4-complex, O:12.17, O:15, O:18, O:19, O:21, O:21.38, O:22, O:24, O:48 and O:51 and these were detected in wildlife only, while the serotypes O:38 and O:41 also were found in the paddock environment but not in the pigs. The serotypes O:2, O:33 and O:52 were found in all three sources.

\subsection{Pulse field gel electrophoresis (PFGE) typing}

C. jejuni isolates of the same serotype from different sources were PFGE-typed for further discrimination between the strains in order to elucidate possible interactions and transmissions between pigs and environment. A total of 13 isolates of serotype O:23.36 from 13 samples representing nine different pigs from paddock $\mathrm{A}$ and $\mathrm{B}$ all yielded an identical 
PFGE pattern, but were different from the PFGE pattern shown by a O:23.36 serotype isolate obtained from a rat. Serotype O:2 was isolated from pigs, environment (soil) and a magpie and an identical PFGE band pattern were found in three of five pigs, one environmental sample and one magpie (Table 2). The remaining four serotype O:2 isolates from two pigs and two environmental samples resulted in four different PFGE band profiles, although the isolates all were obtained from paddock $\mathrm{A}$ in the third trial. The serotype O:1.44 was found in three different pigs and one environmental sample (water) and all showed an identical PFGE type, although the pig isolates were found in two separate trials and paddocks. None of eight serotype 0:33 and O:52 isolates from pigs, environment and wildlife had the same PFGE band patterns. A serotype O:41 isolated from a jackdaw and an environmental sample (water) had dissimilar PFGE band patterns.

\section{Discussion}

This study examined the presence and diversity of thermophilic Campylobacter in organic outdoor pigs and their paddock environment including wildlife caught in the nearby environment. The occurrence of Campylobacter spp. was $100 \%$ in the $8-13$-week-old pigs, and the excretion levels did not seem to decrease within the time-span of this study. Sampling of the pigs was not possible beyond 13 weeks of age, because the Campylobacter investigations were integrated in a bigger study, so it is uncertain if the excretion levels would have decreased at the time of slaughter as observed in conventional pigs (from 4 to $2 \log \mathrm{N} /$ $\mathrm{g}$ faeces) (Weijtens et al., 1999). The organic pigs were not tested before inclusion in the experiment at the age of 8 weeks, but according to other studies, the majority were probably colonized within the first $24 \mathrm{~h}$ (57\% of conventional piglets) (Young et al., 2000) or within the first week ( $75 \%$ of free-range pigs) (Alter et al., 2005).

Campylobacter was isolated from $35 \%$ of the environmental samples, while Campylobacter was found in only $0.7 \%(n=1474)$ of samples from the environment of a conventional farm (Alter et al., 2005). The apparent high exposure from the paddock environment implies that Campylobacter spp. infections in organic pigs might be more persistent, not least due to the difficulties in cleaning of the outdoor paddocks and practicing good hygiene, which is important to reduce infections (Harvey et al., 2000; Weijtens et al., 2000).

A C. jejuni-positive pig was defined as a pig from which $C$. jejuni was obtained at least once irrespective of the isolation method used. Our application of a direct plating approach as well as an enrichment approach for colony-blot hybridization, did not prove any of the approaches to be superior to the other in the detection of $C$. jejuni in pigs, but their combined use increased the overall sensitivity of detection. This has been described in detail elsewhere (Jensen et al., 2005). The detection of $C$. jejuni in $29.8 \%$ of the organic pigs appeared to be high compared to the prevalence of 2.3\% (Boes et al., 2005) and 1.3\% (Anon., 2005) reported for Danish conventional pigs at slaughter. However, the usage of different methods and sampling intensity does not allow a direct comparison of prevalence.

It is common to see a seasonal variation in human campylobacteriosis and broilers with a summer peak in July/August, but a similar variation for conventional pigs has not been reported (Anon., 2005). In this experiment, $C$. jejuni was detected mainly in the third trial (August) but also in the first trial (May), although all pigs originated from the same farm and was born with only 6 weeks interval (Table 1). Furthermore, individual pigs were often $C$. jejuni-positive just once or in non-consecutive weeks and $C$. jejuni was always present in minority to $C$. coli (Table 2 ). This indicated that the presence of $C$. jejuni in a herd or individual pigs may be changing over time and/or that the detection of low numbers of $C$. jejuni in pigs is difficult. Furthermore, it is difficult to conclude whether outdoor pigs generally host more $C$. jejuni than indoor pigs because of their higher environmental exposure to C. jejuni (Kapperud and Rosef, 1983; Petersen et al., 2001; Broman et al., 2002; Brown et al., 2004), which is of concern for outdoor pigs. The detection of $C$. jejuni in conventional herds also seemed independent on the prior $C$. jejuni status (approximately 6 months before) and individual pigs can have a high excretion level of $C$. jejuni but not $C$. coli, which suggests a clumped rather than random occurrence of $C$. jejuni (Boes et al., 2005).

Serotyping of selected strains of $C$. coli also showed that serotypes were inconsistent over time 
and/or that it is difficult to resolve the full picture of serotype diversity if too few isolates are examined (Table 3). For example, up to five different serotypes out of five bacterial isolates were obtained, which is in accordance with the high strain diversity found in pigs in other studies (Weijtens et al., 1997; Moore et al., 2002; Guevremont et al., 2004; Alter et al., 2005; Boes et al., 2005). Even though a certain serotype seemed to dominate in a pig one week, it was not necessarily isolated from this pig the following weeks. In conventional pigs, some subtypes seem to dominate and the genotype pool stabilizes with fewer genotypes with age of the pigs (Weijtens et al., 1997; Moore et al., 2002).

The detection of O:1.44 in three animals seemed special for the organic pigs, as there is no known record of this $C$. jejuni serotype in conventional pigs. From a food safety concern, O:1.44 and O:2 are some of the most common serotypes (38.2\%) among human cases of campylobacteriosis in Denmark (Anon., 2004), while O:23.36, which seem dominant in organic as well as conventional pigs (Nielsen et al., 1997; Anon., 2000; Boes et al., 2005), is less common (3.5\%). The serotypes O:4-complex, O:6.7 and O:11 are also among the top 10 of $C$. jejuni serotypes causing human campylobacteriosis and these serotypes were commonly isolated from the organic pigs, but all isolates were identified as $C$. coli. C. coli with these serotypes are not commonly found in human infections, but these atypical serotypes among $C$. coli strains have previously been found in pigs (Boes et al., 2005). The remaining $C$. coli isolates comprised serotypes similar to findings from conventional pigs (Munroe et al., 1983; Nielsen et al., 1997; Anon., 2003, 2004; Boes et al., 2005). Although some of these C. coli serotypes (e.g. O:30) also have been found in human cases of campylobacteriosis, no epidemiological relationship has been found between pig and human C. coli isolates (Anon., 2004; Guevremont et al., 2004; Siemer et al., 2005).

The pigs seemed to be the likely source of the Campylobacter contamination in the paddock environment, since no Campylobacter was detected before introduction of pigs, no $C$. coli were detected in the wild-life and all $C$. coli serotypes isolated from the paddock environment were also isolated from pigs, but not vice versa (although no PFGE was performed on C. coli). The C. jejuni serotypes found in pigs were also detected in the environment except 0:23.36 despite it was the most frequently isolated serotype in pigs (third trial only) (Table 2). One of five different PFGE genotypes of O:2 seemed consistent in pigs over time and this genotype also occurred in the environment (Table 2). However, $C$. jejuni was isolated from the environment in all three trials and with a higher serotype diversity than in pigs, which indicates a nonpig source. Boes et al. (2005) compared Campylobacter isolates from pigs, cattle and poultry in mixed production herds and indistinguishable $C$. coli genotypes (PFGE) were found in cattle and pigs, while $C$. jejuni shared no genotype between different groups of animals. This indicates a transmission of the $C$. coli from pigs to cattle where $C$. coli normally is rare, but not the other way around for $C$. jejuni. However, no $C$. jejuni was found on 12 farms with pigs only. Identical PFGE genotypes found in grazing cattle and wild birds may originate from a reservoir in the birds, or alternatively, birds may have ingested contaminated cattle faeces (Brown et al., 2004). The role of insects as transmission vectors was not addressed in this study, although potentially important (Hald et al., 2004; Ekdahl et al., 2005). Flies captured at chicken and pig farms, e.g. harboured serotypes that were common in chicken and pigs, respectively (Rosef et al., 1985).

Similar to previous findings (Petersen et al., 2001), numerous serotypes were obtained from the wildlife, but there was only few shared serotypes among environmental and wildlife isolates (O:2, O:33, O:41, O:52). Furthermore, only O:2 shared a PFGE genotype (pigs, paddock environment and a magpie), which indicated a rather low commonality between sources, but to fully address the potential interaction more isolates should be examined. Petersen et al. (2001) suggested wildlife to be a limited reservoir of human infections as only identical PFGE genoypes of O:2 and O:4-complex was found in human, broiler and wildlife (mammals), and Broman et al. (2002) also found only a single identical PFGE type among gull and human isolates.

In conclusion, this study showed a high occurrence (100\%) of Campylobacter spp. in 8-13 weeks old organic pigs raised outdoor with a dominance of $C$. coli. However, the occurrence of $C$. jejuni was high (79\%) under certain but unknown circumstances and included the serotypes (O:2 and O:1.44) that are 
important in human infections. The paddock environment became contaminated with Campylobacter from the pigs, but the diversity of strains indicated other non-pig sources, e.g. the wild fauna. The presence of C. jejuni in the outdoor pigs seemed not strongly related to the exposure from the nearby environment according to the subtype dissimilarities of the majority of the obtained isolates.

\section{Acknowledgements}

We thank Sussi Kristoffersen, Sidsel Boesen and Jonas Michelsen for technical assistance and the animal assistants at the research farm, Rørrendegård for sampling of the pigs. This work was supported by the Danish Research Centre of Organic Farming (DARCOF, II.10), and the Research School for Organic Agriculture and Food Systems (SOAR).

\section{References}

Alter, T., Gaull, F., Kasimir, S., Gurtler, M., Mielke, H., Linnebur, M., Fehlhaber, K., 2005. Prevalences and transmission routes of Campylobacter spp. strains within multiple pig farms. Vet. Microbiol. 108, 251-261.

Anon., 2000. Annual Report on Zoonoses in Denmark 1999. Danish Zoonosis Centre, Danish Veterinary Laboratory.

Anon., 2003. Annual Report on Zoonoses in Denmark 2002. Ministry of Food, Agriculture and Fisheries.

Anon., 2004. Annual Report on Zoonoses in Denmark 2003. Ministry of Food, Agriculture and Fisheries.

Anon., 2005. Annual Report on Zoonoses in Denmark 2004. Denmark, Ministry of Family and Consumer Affairs.

Boes, J., Nersting, L., Nielsen, E.M., Kranker, S., Enoe, C., Wachmann, H.C., Baggesen, D.L., 2005. Prevalence and diversity of Campylobacter jejuni in pig herds on farms with and without cattle or poultry. J. Food Prot. 68, 722-727.

Broman, T., Palmgren, H., Bergstrom, S., Sellin, M., Waldenstrom, J., Danielsson-Tham, M.L., Olsen, B., 2002. Campylobacter jejuni in black-headed gulls (Larus ridibundus): prevalence, genotypes, and influence on $C$. jejuni epidemiology. J. Clin. Microbiol. 40, 4594-4602.

Brown, P.E., Christensen, O.F., Clough, H.E., Diggle, P.J., Hart, C.A., Hazel, S., Kemp, R., Leatherbarrow, A.J., Moore, A., Sutherst, J., Turner, J., Williams, N.J., Wright, E.J., French, N.P., 2004. Frequency and spatial distribution of environmental Campylobacter spp. Appl. Environ. Microbiol. 70, 65016511.

Ekdahl, K., Normann, B., Andersson, Y., 2005. Could flies explain the elusive epidemiology of campylobacteriosis? BMC Infect. Dis. 5,11 .
Guevremont, E., Higgins, R., Quessy, S., 2004. Characterization of Campylobacter isolates recovered from clinically healthy pigs and from sporadic cases of campylobacteriosis in humans. J. Food Prot. 67, 228-234.

Hald, B., Skovgard, H., Bang, D.D., Pedersen, K., Dybdahl, J., Jespersen, J.B., Madsen, M., 2004. Flies and Campylobacter infection of broiler flocks. Emerg. Infect. Dis. 10, 1490-1492.

Harvey, R.B., Young, C.R., Anderson, R.C., Droleskey, R.E., Genovese, K.J., Egan, L.F., Nisbet, D.J., 2000. Diminution of Campylobacter colonization in neonatal pigs reared off-sow. J. Food Prot. 63, 1430-1432.

Harvey, R.B., Young, C.R., Ziprin, R.L., Hume, M.E., Genovese, K.J., Anderson, R.C., Droleskey, R.E., Stanker, L.H., Nisbet, D.J., 1999. Prevalence of Campylobacter spp. isolated from the intestinal tract of pigs raised in an integrated swine production system. J. Am. Vet. Med. Assoc. 215, 1601-1604.

Jensen, A.N., Andersen, M.T., Dalsgaard, A., Baggesen, D.L., Nielsen, E.M., 2005. Development of real-time PCR and hybridization methods for detection and identification of thermophilic Campylobacter spp. in pig faecal samples. J. Appl. Microbiol. 99, 292-300.

Jensen, A.N., Dalsgaard, A., Stockmarr, A., Nielsen, E.M., Baggesen, D.B., 2006. Survival and transmission of Salmonella enterica serovar Typhimurium in an outdoor organic pig farming environment. Appl. Environ. Microbiol. 72, 1833-1842.

Jensen, A.N., Lodal, J., Baggesen, D.L., 2004. High diversity of Salmonella serotypes found in an experiment with outdoor pigs. Wageningen J. Life Sci. 52, 109-117.

Kapperud, G., Rosef, O., 1983. Avian wildlife reservoir of Campylobacter fetus subsp. jejuni, Yersinia spp., and Salmonella spp. in Norway. Appl. Environ. Microbiol. 45, 375-380.

Kramer, J.M., Frost, J.A., Bolton, F.J., Wareing, D.R., 2000. Campylobacter contamination of raw meat and poultry at retail sale: identification of multiple types and comparison with isolates from human infection. J. Food Prot. 63, 1654-1659.

Madden, R.H., Moran, L., Scates, P., 2000. Optimising recovery of Campylobacter spp. from the lower porcine gastrointestinal tract. J. Microbiol. Meth. 42, 115-119.

Manser, P.A., Dalziel, R.W., 1985. A survey of Campylobacter in animals. J. Hyg. 95, 15-21.

Mikkelsen, L.L., Naughton, P.J., Hedemann, M.S., Jensen, B.B., 2004. Effects of physical properties of feed on microbial ecology and survival of Salmonella enterica serovar Typhimurium in the pig gastrointestinal tract. Appl. Environ. Microbiol. 70, 34853492.

Moore, J.E., Lanser, J., Heuzenroeder, M., Ratcliff, R.M., Millar, B.C., Madden, R.H., 2002. Molecular diversity of Campylobacter coli and $C$. jejuni isolated from pigs at slaughter by flaARFLP analysis and ribotyping. J. Vet. Med. B: Infect. Dis. Vet. Public Health 49, 388-393.

Munroe, D., Prescott, J.F., Penner, J.L., 1983. Campylobacter jejuni and Campylobacter coli serotypes isolated from chickens, cattle, and pigs. J. Clin. Microbiol. 18, 877-881.

Nielsen, E.M., Engberg, J., Madsen, M., 1997. Distribution of serotypes of Campylobacter jejuni and C. coli from Danish patients, poultry, cattle and swine. FEMS Immunol. Med. Microbiol. 19, 47-56. 
Penner, J.L., Hennessey, J.N., Congi, R.V., 1983. Serotyping of Campylobacter jejuni and Campylobacter coli on the basis of thermostable antigens. Eur. J. Clin. Microbiol. 2, 378-383.

Petersen, L., Nielsen, E.M., Engberg, J., On, S.L., Dietz, H.H., 2001. Comparison of genotypes and serotypes of Campylobacter jejuni isolated from Danish wild mammals and birds and from broiler flocks and humans. Appl. Environ. Microbiol. 67, 3115-3121.

Ribot, E.M., Fitzgerald, C., Kubota, K., Swaminathan, B., Barrett, T.J., 2001. Rapid pulsed-field gel electrophoresis protocol for subtyping of Campylobacter jejuni. J. Clin. Microbiol. 39, 18891894.

Rosef, O., Kapperud, G., Lauwers, S., Gondrosen, B., 1985. Serotyping of Campylobacter jejuni, Campylobacter coli, and Campylobacter laridis from domestic and wild animals. Appl. Environ. Microbiol. 49, 1507-1510.

Siemer, B.L., Nielsen, E.M., On, S.L., 2005. Identification and molecular epidemiology of Campylobacter coli isolates from human gastroenteritis, food, and animal sources by amplified fragment length polymorphism analysis and Penner serotyping. Appl. Environ. Microbiol. 71, 1953-1958.

Totten, P.A., Patton, C.M., Tenover, F.C., Barrett, T.J., Stamm, W.E., Steigerwalt, A.G., Lin, J.Y., Holmes, K.K., Brenner, D.J., 1987.
Prevalence and characterization of hippurate-negative Campylobacter jejuni in King County, Washington. J. Clin. Microbiol. 25, 1747-1752.

Waldenstrom, J., Broman, T., Carlsson, I., Hasselquist, D., Achterberg, R.P., Wagenaar, J.A., Olsen, B., 2002. Prevalence of Campylobacter jejuni, Campylobacter lari, and Campylobacter coli in different ecological guilds and taxa of migrating birds. Appl. Environ. Microbiol. 68, 5911-5917.

Weijtens, M.J.B.M., Reinders, R.D., Urlings, H.A.P., van der Plas, J., 1999. Campylobacter infections in fattening pigs; excretion pattern and genetic diversity. J. Appl. Microbiol. 86, 63-70.

Weijtens, M.J.B.M., Urlings, H.A.P., van der Plas, J., 2000. Establishing a Campylobacter-free pig population through a topdown approach. Lett. Appl. Microbiol. 30, 479-484.

Weijtens, M.J., van der Plas, J., Bijker, P.G.H., Urlings, H.A.P., Koster, D., van Logtestijn, J.G., Huis in't Veld, J.H.J., 1997. The transmission of Campylobacter in piggeries; an epidemiological study. J. Appl. Microbiol. 83, 693-698.

Young, C.R., Harvey, R., Anderson, R., Nisbet, D., Stanker, L.H., 2000. Enteric colonization following natural exposure to Campylobacter in pigs. Res. Vet. Sci. 68, 75-78. 